\title{
CONCEPTUAL MODELING OF LARGE WEB SITES
}

\author{
Bernhard Strauch, Robert Winter \\ Institute of Information Management, University of St. Gallen \\ Mail: Mueller-Friedberg-Strasse 8, CH-9000 St. Gallen (Switzerland) \\ E-mail: firstname.lastname@unisg.ch \\ Phone: +41712242935 Fax: +41712242936
}

\begin{abstract}
Current web site development is still dominated by technical issues. In order to enable efficient communication between developers and to provide a stable foundation for adopting new technologies, web sites should be derived from conceptual models. Based on the state-of-the-art of conceptual modeling as implemented in current CASE environments as well as web site development tools, the "essence" of a web site is identified, and an adequate conceptual meta model is proposed. Appropriate web site models are intended to capture not only hierarchical document structure and hypertext semantics, but also dynamical page generation from databases as well as explicit and implicit navigation. It becomes evident that web sites can be regarded as supersets of traditional information systems, thereby requiring conceptual modeling to include various additional features. The proposed meta model comprises several classes of information objects, various types of associations, design rules, and quality checks. For illustration purposes, the model is applied to an existing web site. Commercial web site development tools are analyzed with regard to the extent to which they support conceptual web site modeling.
\end{abstract}

\section{INTRODUCTION}

Early systems development was dominated by using authoring tools ("editors") to manually edit procedural program code. As a consequence, hand-written code mixing up data usage and functional aspects was difficult to maintain [10]. Besides of expensive quality control and communication problems among developers, the resulting code suffered from various implementation dependencies, thereby forcing developers to re-do large portions of the development process when technical details (e.g. file structures, access paths) change. A rather similar approach can be observed when looking at today's web site development practice [14]. By creating web sites using HTML authoring tools, complex code is created that does not only mix up appearance and contents, but also depends widely on implementation details. Moreover, the utilization of different authoring tools complicates communication between developers. As an example, the following problems usually occur with regard to navigation when different web sites have to be integrated:

- Navigation is interpreted and implemented in different ways depending on the authoring tool in use. Different tools use identical terms for different concepts or different terms for identical concepts.

- Navigation is not based on user requirements for optimal access to information objects or associations between information objects (see e.g. [12][13]). Instead, implementation details like various frame variants dominate design.

- As a consequence, similar navigational concepts are implemented (and specified) in different ways so that explicit integration efforts are necessary to identify common structures and implement them consistently.

In order to enable efficient communication between developers and to provide a stable foundation for adopting new technologies, conceptual modeling of web sites is essential. We understand web sites as server components of distributed applications which use the HTTP protocol to exchange data between servers and clients ("browsers"). By this definition, the principal problem of web site development becomes apparent: Even the relevant class of application components is defined by technical attributes (HTTP protocol, server functionality) instead of conceptual differences. Conceptual modeling should be independent of all technical and application dependent details. Before analyzing conceptual issues in web site design, proposing an appropriate conceptual model, and checking support potentials of current web site development tools, therefore, we should discuss whether our 
initial definition is appropriate, i.e. to what extent web sites conceptually differ from traditional information systems.

\section{Web sites vs. traditional information systems}

Traditional business applications support business processes by implementing data entry and data manipulation of business transactions. Web applications go beyond this functionality by integrating different media for information representation and by hypertext functionality, thereby supporting not only the handling of business transactions, but also the storage and transfer of knowledge.

As a consequence, not only organizational, functional, and data views of business processes have to be specified during conceptual modeling. In addition, complex knowledge components and their associations should be specified to enable flexible access and efficient navigation.

As an example, the data view of traditional information systems development and web site development is compared: A traditional data model comprises types of structured information objects (e.g. order, customer, product) along with their attributes, types of relationships between information object types (e.g. generalization dependencies, existential dependencies), and consistency constraints. All modeling is done on a type level.

Of course, all of these elements types can be found in the data model of a web site. But a web site representing complex knowledge may also comprise the following additional element types:

- $\quad$ arious one-of-a-kind information objects (i.e. single object instances not belonging to any object type, e.g. a mission statement)

- $\quad$ various types of unstructured information objects (e.g. documents), and

- additional types of associations between information objects (e.g. "part-of" relationships used for structuring a knowledge domain and "association" relationships used for navigation within a knowledge domain).

\section{Related work and overview}

The lack of conceptual structures in hypertext documents has been addressed long before the World Wide Web emerged: "Without additional structuring mechanisms often very large, global and flat hypermedia networks (or hyperdocuments) are created which are hard to understand for readers. Users often become disoriented and a carefully directed search for a particular information object is more complicated in such networks." [9] The HYDESIGN model [9] proposes classes of (hypermedia) objects comprising "atomic nodes", "SBL nodes", and links between those nodes. Atomic nodes inherit general class properties and represent content. SBL (Structure Behavior Locality) nodes represent additional information: Structure defines how nodes and links can be connected, behavior defines the dynamics of nodes and links, and locality defines a "local" environment, i.e. a submodule of the hypermedia network. By this approach, important conceptual constructs like navigation paths or aggregate objects (= sub-networks) are introduced into hypermedia design. When hypermedia design approaches (e.g. [4][5][6][9]) are used for the conceptual design of web sites, however, important aspects like common standards of web navigation, interface design, conceptual differences between links, and overall site design are not taken into account.

Since many problems addressed by hypermedia design like "disorientation" or "cognitive overhead" are also prevailing in large web sites, however, the basic approach of a hierarchical, recursive model of node classes and link classes is useful for web site design. But in addition to static links between particular documents, common standards of web navigation imply dynamic links: "Dynamic node-link binding has greater potential for increasing the flexibility and maintainability of a hypermedia network." [3] In [4], nodes ("information chunks") are complemented by "anchors" that define which components of a node are linked to other nodes.

With the widespread use of the World Wide Web, many dedicated approaches to conceptual design of web sites have been proposed (e.g. [7][8]). Most approaches, however, either do not cover dynamic generation of web sites from databases and experience from traditional information systems design which are crucial for engineering very large web sites, or do not separate technical issues from conceptual modeling.

According to W3DT (initially called SHDT) [2][16], the development process comprises seven steps, guiding the designer from requirements analysis to implementation of the web site. For the first phase (requirements analysis), a collection of methods to determine user requirements is provided. The design phase is divided into information structuring, navigational design, organizational design, and interface design. Information structuring and navigational design is understood as an iterative mental process where a large knowledge domain has to be organized and structured into information objects, which are then linked and evaluated. Organizational design assigns information on maintenance and organizational integration to the information objects. When the design phase is completed, the information objects are implemented by HTML pages and gateway scripts, and the access structures of the model are implemented by links within the WWW site. 
The W3DT research group has shown that it is possible to capture the navigational structure of real-world web sites. In W3DT's meta model, a web site is composed of pages, links, and layouts. While layout is not further specified, two different types of links and four different types of pages are differentiated. This simple classification, however, is not sufficient to capture the increased complexity of current HTML pages. Moreover, it is not differentiated between page templates and page contents.

Following this introduction, we try to identify the implementation independent specification ("essence" [11]) of a complex web site. In section 3, an appropriate meta model is proposed that captures not only hierarchical document structure and hypertext semantics, but also allows for an implementation by dynamical page generation from databases and using various approaches to explicit and implicit navigation. Basic design rules for the proposed conceptual model are presented in section 4 . In section 5, current web site development tools are analyzed with regard to extent to which conceptual web site modeling is supported.

\section{THE "ESSENCE" OF A WEB SITE}

The benefits of conceptual modeling result from creating stability: Often contents change, but the general page structure remains unchanged. At other occasions, templates change, but contents remain stable. For conceptual modeling of web sites, therefore, content should be separated from navigational structure, navigational structure should be separated from layout (i.e., general page structure), and layout should be separated from content.

But if all implementation-related specifications are to be neglected, what is then the essence of a web site? As for traditional applications, organizational, functional, and data aspects of business transactions have to be specified. In addition, representation and access aspects of complex information areas have to be specified by means of

- (structured and non-structured) information objects,

- "part-of" (i.e. hierarchical) relationships between information objects, and

- "associative" (i.e. non-hierarchical) relationships between information objects.

Information objects may be one-of-a-kind instances that do not match any type definition, or may be instances of some type of information objects. They may be administrated internally (i.e. within the same organizational context as the web site) or externally. They may be implemented by operating system files, file records, tuples of relational tables, or mail accounts.

Hierarchical relationships may be implemented by file system structure, HTML navigation, or by applets. Nonhierarchical relationships may be implemented by hyperlinks, mailtos, or downloads.

Information objects may be complex, i.e. may comprise several components. As a consequence, we have to represent

- components of information objects (e.g. character strings, pictures, textual tables) and

- hierarchical relationships between information objects and their components.

Information object components are usually implemented by operating system files or tuples of relational tables. Hierarchical component links may be implemented by physical inclusion, as reference using frame or border techniques, or using style sheets.

\section{Why should information objects and their components be differentiated?}

The easiest conceptual interpretation of a web site is a multi-stage "assembly" of elementary information objects. However, information objects should be differentiated from their components: Information object contents change often. Since conceptual models should provide some basic amount of stability, it is necessary to differentiate a stable and a volatile part in the "assembly" structure of a web site. Information objects denote the borderline between the volatile and the stable portion of the network: While their contents change often (which has to be reflected in updating components and/or updating hierarchical component structure), their conceptual representation is stable so that the "upstream" portion of the web site remains unchanged.

E.g., status, employee assignments, schedule, available documents, and other attribute-like properties of a project change quite often, while its organizational embedding and its links to other projects remain quite stable. As a consequence, project documents should be regarded as information object components, while the project itself should be regarded as an information object.

In systems implementation, the differentiation of information objects from their components is reflected making the former accessible by hyperlinks, while the latter can not be separately accessed. 
Information object components are the building block of every web site. Information objects can be identified as simplest information component aggregates that guarantee some degree of stability. Navigation areas are those portions of a web site which are so tightly coupled that some degree of navigation traffic is to be expected. Web sites are those portions of the World Wide Web whose contents can be updated in some organizational context. The resulting conceptual structure of the World Wide Web can be interpreted as a five level hierarchy. The web site level, the navigation area level, and the component level may comprise several sub-levels to reflect organizational responsibilities (web site level), relationships between information objects (navigation area level), and information object structure (component level).

\section{CONCEPTUAL MODEL}

Based on the discussion in section 2, the various types of elements of the conceptual model are presented in this section.

\section{- Components of information objects}

- $\quad$ Structured classifiable components

This class of components represents information that can be regarded as an instance of some generalized class of information with certain attributes. E.g., data about a person's publications are structured because every publication has certain attributes and are classifiable because each data is an instance of a common class "publication". For structured classifiable components, structure and contents can be separated.

\section{- Unstructured classifiable components}

This class of components represents information that can be regarded as an instance of some generalized class of information, but has no common attributes within that class. E.g., job descriptions may not follow some formal structure, but are nevertheless classifiable because each job description is an instance of a common class "job description". For unstructured classifiable components, structure cannot be separated from contents.

- $\quad$ Structured, one-of-a-kind components

This class of components represents information that does not belong to some generalized class of information, but comprises certain attributes. E.g., a form is a structured, one-of-a-kind component because every form has certain attributes (layout, boilerplate texts, field names, etc.), but cannot be assigned to some common meaningful class. For structured one-of-a-kind components, structure and contents can be separated.

- $\quad$ Unstructured, one-of-a-kind components

This class of components represents information that neither belongs to some generalized class of information nor has certain attributes. E.g., a mission statement is an unstructured, one-of-a-kind component because only one such text exists within a web site, and no general statement structure is available. For unstructured one-of-a-kind components, structure cannot be separated from contents.

\section{- Information objects}

- (Internal) Informational objects

Information objects are the simplest information component aggregates that guarantee some degree of stability.

\section{- $\quad$ Navigators}

Navigators are application components that allow users to efficiently navigate within some portion of the web site. In contrast to ordinary information objects, navigators represent informational structure instead of the information itself. However, navigators are composed of several components so that they represent a subclass of information objects.

\section{- External information objects}

External information objects are not part of a web site, but can be accessed from that web site, e.g. by hyperlinks. In contrast to internal information objects, the structure of external information objects is unknown. At most, some basic structural information can be generated from the URL extension (HTML, IDC; HTX, ASP, NSF, CGI, etc.). 


\section{- Relationships}

- Hierarchical relationships

Hierarchical relationships represent "part-of" links between internal information objects. Navigators usually are based on hierarchical relationships.

- $\quad$ Associative relationships

Associative relationships represent associations between information objects that are not related hierarchically or by navigators.

- Navigational relationships

Navigational relationships represent associations between (internal and external) information objects and navigators. In contrast to hierarchical and associative relationships that are used to represent information semantics, navigational relationships represent the application interface are and used to support the browsing process [12].

- $\quad$ Component relationships

Component relationships represent the structure of internal information objects. In contrast to hierarchical and associative relationships, component relationships do not imply any hypertext functionality.

\section{Notation}

We denote the element types of the conceptual model as follows:

- Components of information objects

\begin{tabular}{|c|c|c|}
\hline & Classifiable & One-of-a-kind \\
\hline 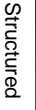 & & \\
\hline 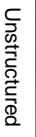 & & \\
\hline
\end{tabular}

- Information objects

(Internal) information object

Navigator

External information object

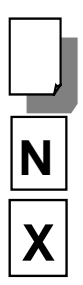

- Relationships

Hierarchical relationship

Associative relationship

Navigational relationship

Component relationship

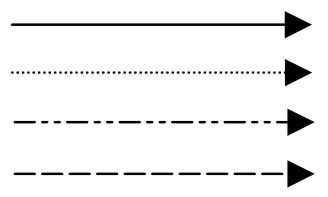

Figure 1 illustrates the application of the proposed model to an existing web site of an university workgroup. The levels are related to hierarchical relationships within the information objects of the web site. 


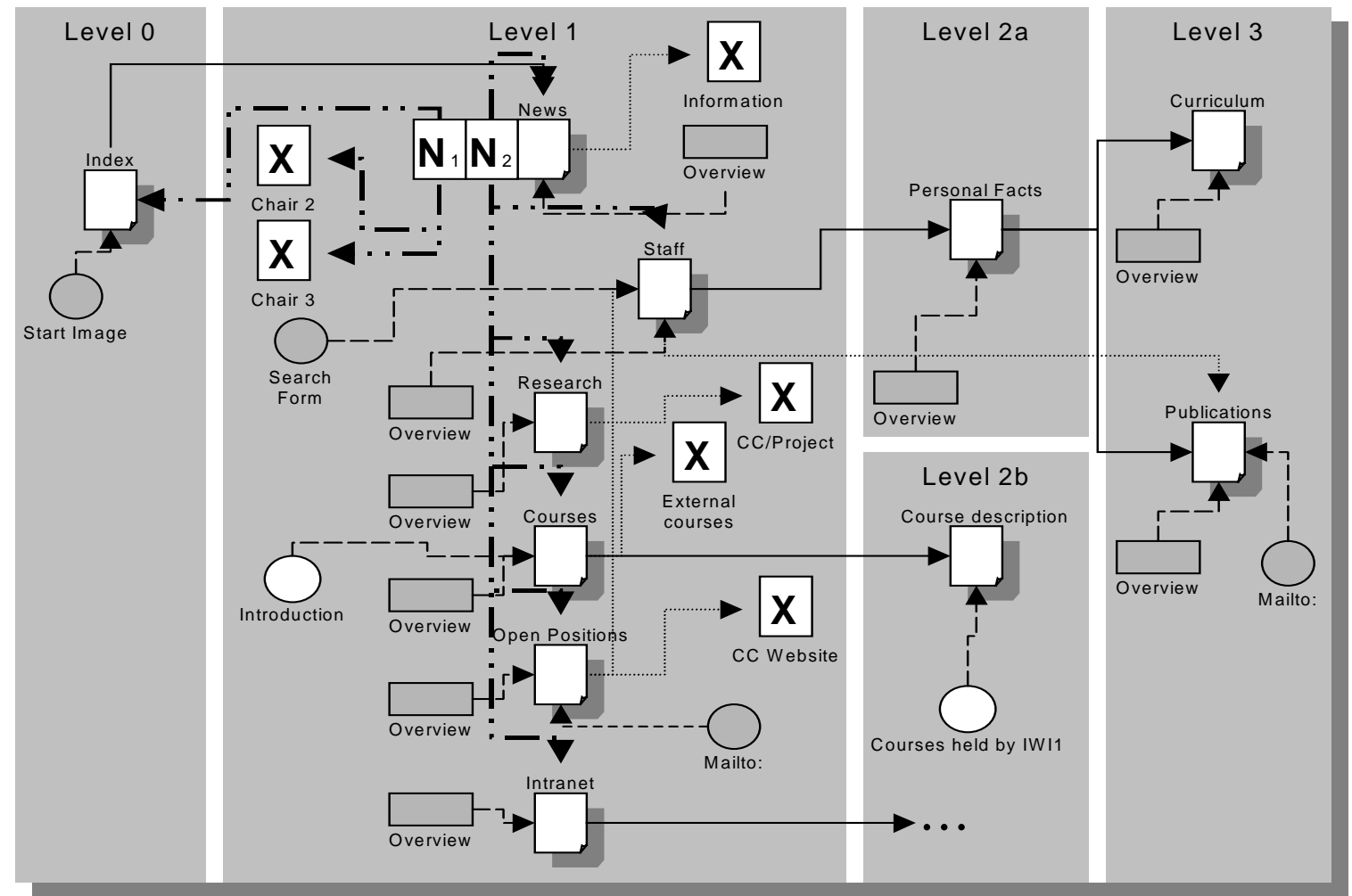

Figure 1. Example conceptual web site schema

\section{Quality}

The proposed conceptual model implies the following quality controls:

Within the web site

- All hierarchical relationships must link an internal information object to one or more internal information objects

- All associative relationships must link an internal information objects to one or more internal or external information objects

- All navigational relationships must link a navigator to one or more internal or external information objects

- All component relationships must link an internal information object to one or more components

Within the World Wide Web

- All URLs referenced by associative or navigational relationships must exist

\section{DESIGN RULES}

For conceptual modeling of a web site, model elements, graphic notation, and quality controls have to be complemented by some basic methodology, i.e. at least by basic design rules and a recommendation of a certain sequence of design phases. Based on our experience with web site design and the discussion of essential web site elements, we propose the following task sequence:

\section{(1) Identification of information objects}

Whether an information representation is an information object or should be regarded as a component of an information object should be decided by analyzing its update behavior: If frequent updates can be expected not only for its contents, but also for its relationships, it should be represented as a component. If updates will mostly be restricted to contents, it may be represented as an information object.

\section{(2) Creation of hierarchical structure}

In contrast to associative relationships, hierarchical relationships create a consistent directed graph of information objects. Based on the hierarchy, levels of the conceptual web site schema can be identified (see figure 1). All hierarchical relationships link an information objects on level $n$ to one or more information 
objects on level $n+1$. Since hierarchical relationships help to structure the knowledge domain to be modeled, conceptual modeling should start with building hierarchical relationships.

\section{(3) Creation of associative structure}

All semantic links that do not represent "part-of" relationships are represented by associative relationships in the next step. Associative relationships are bound to certain levels of the schema. External information objects are always linked to internal information objects by associative relationships. They should be modeled on the same schema level as the internal information objects they are linked to.

\section{(4) Design of navigators}

Although usually being based on hierarchical or associative relationships, navigators and navigational relationships represent separate concepts. While the navigator itself represents application interface properties, navigational relationships represent its behavior. Using navigators, application users can easily navigate in a knowledge domain without having to follow hierarchical or associative relationships.

One or more navigators can be directly linked to an information object. If more than one navigator is linked to an information objects, an index number is introduced. A navigator is linked to that information object whose browsing shall initially create the navigation menu. A navigator it "inherited" by a set of other information objects on the respective schema level and on the next schema level. By separating schema levels according to information object sets related to navigators, elements of complex web site schemas can be arranged.

\section{(5) Representation of components}

For every information object, component relationships and components can be represented in the web site schema or (for complex schemas) in a detail subschema for each information object. Since relationships (including navigational relationships) are defined between information objects only, modifications of component structure do not affect the overall schema. Components may be reused by more than one information object.

\section{TOOL SUPPORT}

In this section, commercial end-user development tools for web sites are discussed with regard to conceptual modeling. Unlike most tools that do not support for conceptual modeling at all, Microsoft's Frontpage 98 and Macromedia's Dreamweaver have appropriate features and are discussed in the following. Since Microsoft Visual Interdev 6.0's features are very similar to Frontpage 98, this tool is not being discussed separately. Adobe PageMill 2.0 for Windows is basically a HTML editor with WYSIWIG capabilities, i.e. without an explicit conceptual model. However, Adobe claims [1] that the Macintosh version has some modeling features like a "site view" and an "external reference view". Interleaf's Jamba is a generator for web pages that include multimedia objects implemented by Java code. Applets can be generated without any knowledge of the Java syntax by using a graphical interface for the assembly of multimedia objects to pages. As building blocks, a large number of buttons, graphics, menus, text fields, and links are provided. However, Jamba is restricted to the development of single pages (not complete sites) without extended procedural features and without support for requirements specification, analysis, or design.

\section{Microsoft FrontPage 98}

Frontpage 98 provides only one type of information object which is directly corresponding to HTML files stored on the web server. Component structure cannot be modeled. Neither navigators nor external information objects can be differentiated. Navigators, however, are automatically generated. But these structures depend on Frontpage's navigational model and properties that have to be coded without any conceptual representation. For each web site, the designer has to decide globally whether borders should appear or not.

Hierarchical relationships between information objects can be modeled as "navigational model" (see figure 2). Associative relationships, however, as well as navigational relationships in our definition and component relationships are unknown. When updating hierarchical relationships, the deletion of a single relationship may trigger the deletion of an entire sub-tree including the respective information objects.

Only a few basic concepts of conceptual web site design are currently supported by Frontpage 98. It is necessary to extensively re-code web sites generated by Frontpage 98 to allow for features like associative links, external links, component reuse, non-standard navigators, etc. Obviously, respective web sites are not really based on a stable conceptual schema so that technical innovation will require significant development efforts. 


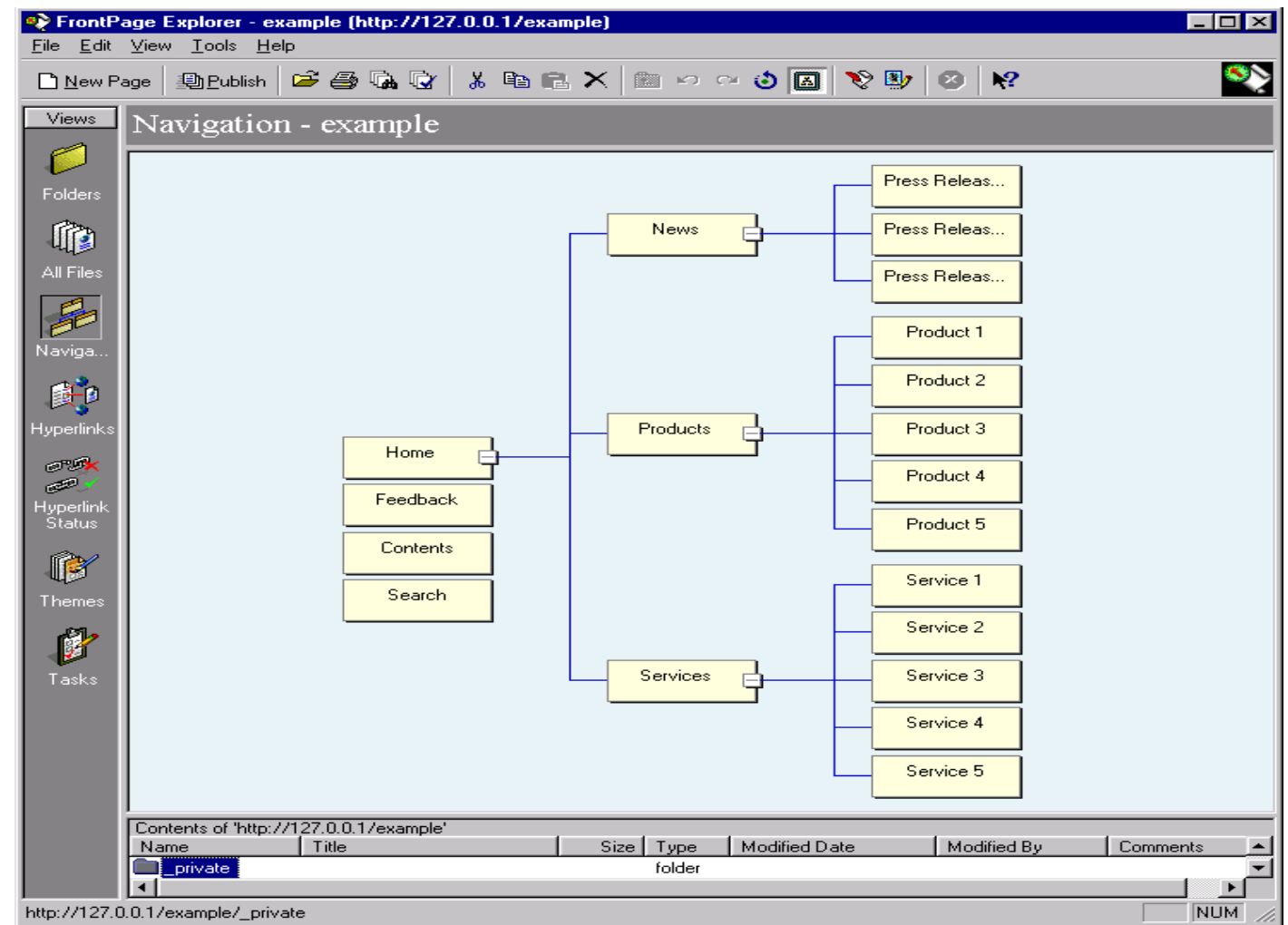

Figure 2. Web site schema in Frontpage 98

\section{Macromedia Dreamweaver 2.0}

In Dreamweaver's conceptual model, two classes of information objects exist. On the one hand, there are information objects which represent a physical HTML file on the web server. On the other hand, there are information objects like external links, mailto tags, and images. To some extent, these two classes correspond to information objects (files) and components (links, images) in our model (see figure 3). However, the latter group of objects is not differentiated so that meaningful quality checks or design rules cannot be applied.

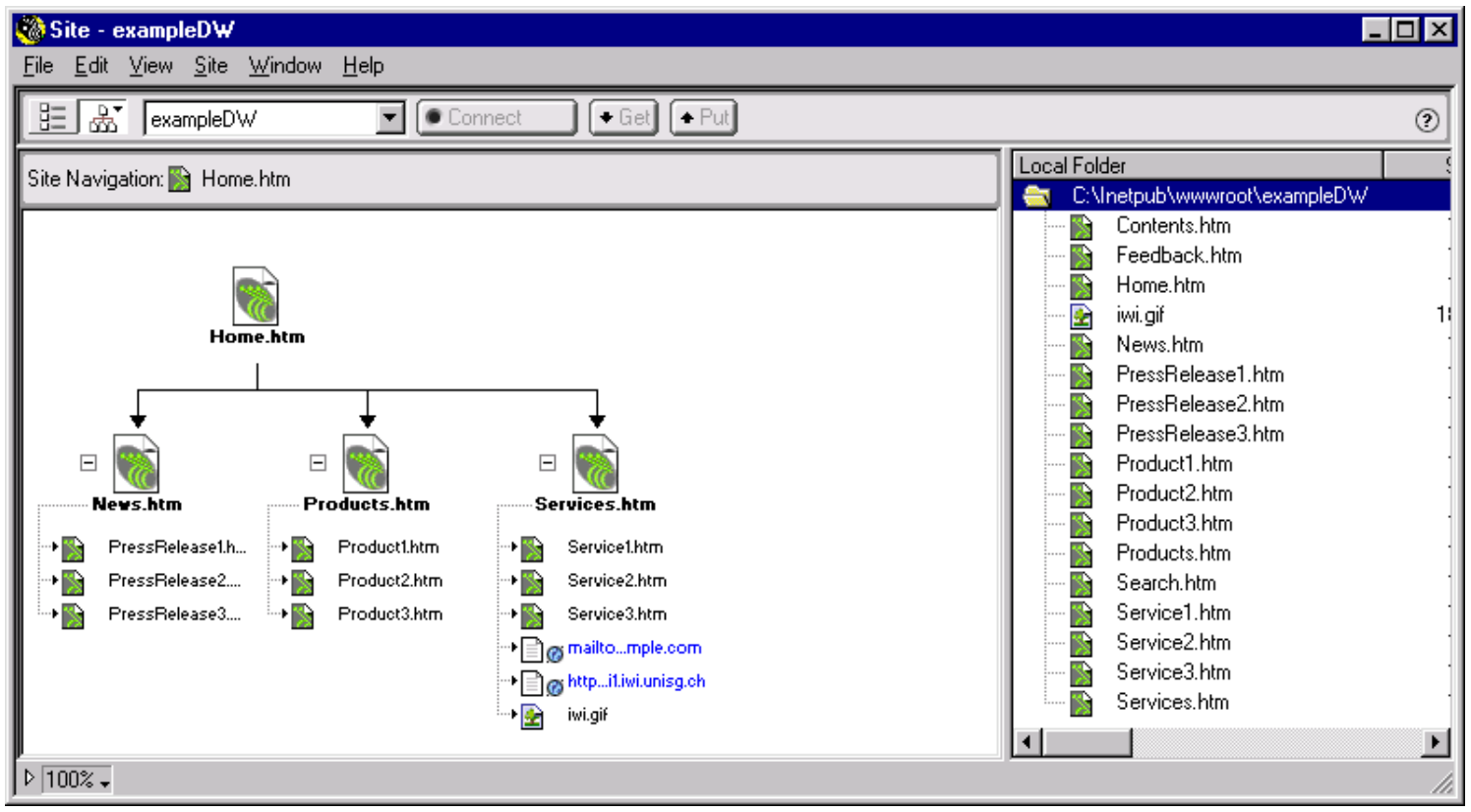

Figure 3. Web site schema in Dreamweaver 2.0 
Between information objects, hierarchical relationships as well as associative relationships can be modeled. However, Dreamweaver does not provide a different notation for these two classes of relationships. Although associative links can be modeled, web sites can only be displayed as expandable trees (based on hierarchical relationships) and not as networks that also include associative relationships.

Dreamweaver does not support the design of navigators and navigational relationships. The generator directly creates HTML hyperlinks from associative relationships.

\section{CONCLUSIONS}

Based on a short discussion of related work and an analysis of the essence of a web site, a conceptual model is proposed that allows for the specification of useful quality checks, design rules, schema arrangement rules, and schema documentation for web sites. Most of the proposed features, however, are currently not supported by commercial web site design tools.

In order to prevent current web site development to create the legacy systems of the future, it is necessary to derive web-based information systems from a stable conceptual foundation. In order to provide a dependable specification for web design software vendors, approaches to conceptual modeling from scientists and practitioners have to be integrated, thereby taking into account findings from hypertext design as well as experience from traditional CASE practice. Hopefully, specific discussion of web site modeling issues will help such specifications to emerge.

\section{REFERENCES}

[1] Adobe Systems Inc. (1997) Adobe PageMill Details - SiteMill for Macintosh. <http://www.adobe.com/ prodindex/pagemill/siteben.html> (27 Nov 1997).

[2] BICHLER M and NUSSER S (1996) SHDT-The Structured Way of Developing WWW-Sites, Proceedings of the 4th European Conference on Informa-tion Systems (DIAS COELHO J et al., eds.), pp. 1093-1101.

[3] CHEN C (1997) Structuring and Visualising the WWW by Generalised Similarity Analysis. In Proceedings of the eighth ACM conference on Hypertext HYPERTEXT'97, pp. 177-186.

[4] DE BRA P and HOUBEN G J (1992) An Extensible Data Model for Hyperdocuments. Proceedings of the ACM conference on Hypertext ECHT'92, pp. 222-231.

[5] DIÁZ A, ISKOWITZ T, MAIORANA V, and GILABERT G (1995) RMC: A Tool To Design WWW Applications. <http://www.stern.nyu.edu/ tisakowi/ papers/www-95/rmcase/187.html> (27 Nov 1997).

[6] ISAKOWITZ T, STOHR E A, and BALASUBRAMANIAN P (1995) RMM: A Methodology for Structured Hypertext Design. Communications of the ACM 38 (8), pp. 34-44.

[7] LYARDET F D, MERCERAT B, and MIATON L (1999) Putting Hypermedia Patterns to Work: a Case Study. <http://pelican.info.unlp.edu.ar/ht992/patternsAtWork.html> (10 May 1999)

[8] LYNCH P J and HORTON S (1999) Web Style Guide: Basic Design Principles for Creating Web Sites. <http://info.med.yale.edu/caim/manual/contents.html> (10 May 1999)

[9] MARMANN M and SCHLAGETER G (1992) Towards a Better Support for Hypermedia Structuring: The HYDESIGN Model. Proceedings of the ACM conference on Hypertext ECHT'92, pp. 232-241.

[10] MARTIN J (1982) Application development without programmers, Englewood Cliffs, Prentice-Hall.

[11] McMENAMIN S M and PALMER J F (1984) Essential Systems Analysis, New York, Yourdon Inc.

[12] MORVILLE P and ROSENFELD L (1998) Designing Navigation Systems <http://webreview.com/wr/pub/98/02/20/arch/index.html> (29 Apr 1999)

[13] RICHMOND A (1999) Navigation Architecture of The WDVL, <http://www.stars.com/WebRef/Navigation/WDVL.html> (28 Apr 1999)

[14] ROSENFELD L and MORVILLE P (1998) Information Architecture for the World Wide Web - Designing Large-scale Web Sites, O'Reilly \& Associates.

[15] SCHRANZ M (1998) Engineering Flexible World Wide Web Services. In Proceedings of the Symposium on Applied Computing 1998 (CARROLL J et al., eds.), pp. 712-718.

[16] W3DT-TEAM (1996) W3DT - WWW Design Technique <http://wwwi.wu-wien.ac.at/ w3dt/> (26 Nov 1997). 